\title{
Las Tecnologías de la Información y la Comunicación (TICs) en la Enseñanza-Aprendizaje de la Química Orgánica a través de Imágenes, Juegos y Videos
}

\author{
María R. Hernández, Verónica M. Rodríguez, Francisco J. Parra y Pedro Velázquez \\ Universidad de Guadalajara, CUCEI, Depto. de Química, Blvd. Marcelino García Barragán \#1421, esq. \\ Calzada Olímpica, C. P. 44430 Guadalajara, Jalisco-México. (e-mail: mraquelh03@hotmail.com, \\ veromrb@yahoo.com, fjparra58@hotmail.com,copony01@hotmail.com)
}

Recibido Abr. 30, 2013; Aceptado Jun. 17, 2013; Versión final recibida Oct. 2, 2013

\begin{abstract}
Resumen
Se presentan resultados de un trabajo docente, efectuado con el objetivo de que nuestros alumnos de primer año de universidad adquirieran, de forma sencilla y efectiva, conocimientos de Química Orgánica básica. Las herramientas educativas utilizadas para la enseñanza de conceptos teóricos simples y complejos, están sustentadas en el uso de las Tecnologías de Información y Comunicación (TICs). Se elaboró material didáctico visual a color en 2D y 3D, juegos y videos educativos, todos accesibles en formato electrónico. Se ha encontrado que con el uso de material visualmente ilustrativo se facilitó y enriqueció el aprendizaje. Como resultado de esto, hubo un incremento en el porcentaje de aprobados y el promedio de las calificaciones. Esto produjo un impacto positivo en la eficiencia terminal del curso por lo que la metodología de enseñanza mostró ser de gran utilidad.
\end{abstract}

Palabras clave: química, orgánica, enseñanza, aprendizaje, TICs

\section{Information and Communication Technologies (ICTs) for the Teaching of Organic Chemistry by Means of Pictures, Games and Videos}

\begin{abstract}
We report results of a teaching method applied with the aim that our freshmen students could acquire, in a simple and effective way, knowledge of basic organic chemistry. The educational tools used for the teaching of simple and complex theory concepts are supported by the use of the information and communication technologies (ICT). Visual, in color, didactic material was elaborated in 2D and 3D, as well as games and educational videos, all accessible in electronic format. It was found that, with such approach, consisting of illustrative visual material, the learning experience was enhanced and facilitated. Furthermore, the passing rate and the grades of the students increased. This caused a positive impact over the efficiency of the school program, which showed that the teaching methodology was very useful.
\end{abstract}

Keywords: organic, chemistry, teaching, learning, ICT 


\section{INTRODUCCIÓN}

En el Centro Universitario de Ciencias Exactas e Ingenierías de la Universidad de Guadalajara se imparte la materia de Química Orgánica I, obligatoria y de tronco común para las carreras de QuímicoFarmacobiólogo, Licenciatura en Química e Ingeniería Química. Debido al tiempo reducido para cubrir los programas de estudio, y a que la enseñanza tradicional (pizarrón o pizarra blanca).contraviene con el "mundo real" en el que los alumnos viven, porque han cambiado sus hábitos de estudio, y la forma de interrelacionarse como resultado de las experiencias con la tecnología fuera de la escuela (Hernández, 2003), se hacen necesarias nuevas formas de enseñanza (Bekerman y Dankner, 2010).

Se ha comprobado que los jóvenes actuales tienen más desarrollado el canal visual, y una alta estima hacia lo lúdico, debido al tiempo que han sido expuestos a las nuevas tecnologías de la comunicación, desde temprana edad (Moreno, 2006). Así mismo, se ha demostrado que en el aprendizaje de la Química, la cognición visoespacial es de vital importancia (Wu y Shah, 2004). Aunado a lo anterior, los estudiantes quieren crear usando las herramientas de su tiempo, trabajar con sus compañeros tomando sus propias decisiones y compartiendo el control, no sólo en su clase sino también conectándose con otros jóvenes alrededor del mundo para compararse y competir entre ellos; además quieren que los conocimientos adquiridos sean aplicados inmediatamente en algo real (Prensky, 2010). En el nuevo paradigma educativo es relevante el aprendizaje a través del trabajo cooperativo, en el cual los sistemas multimedia proporcionan valiosas oportunidades, tanto en el aula como por internet (Jiménez y Llitjós, 2006).

Las "TICs" (Tecnologías de la Información y la Comunicación) agrupan elementos y técnicas usadas en el tratamiento y transmisión de información; son herramientas que pueden ser aprovechadas para la construcción de material didáctico, facilitando el aprendizaje y el desarrollo de habilidades (Carnoy, 2004), al requerir que el alumno movilice su pensamiento crítico y analítico, mientras interactúa con ellas (Miranda et al., 2012), desarrollando su capacidad para responder a los desafíos y problemas implicados en cada materia y en su futuro laboral. Las "TICs" se están convirtiendo en instrumentos habituales en las escuelas públicas de los países desarrollados, extendiéndose hasta los sistemas educativos de los países en vías de desarrollo (Khvilon E., et al., 2004). Estas tecnologías permiten, que los procesos cognitivos tengan un mayor papel en el aprendizaje de materiales educativos y científicos complejos (como sucede en el campo de la Química que además de su contenido científico, puede aportar un cierto sentido artístico; basta revisar la literatura química actual para descubrir esto) respondiendo a la "evolución" natural de los métodos de aprendizaje y a la interrelación de los jóvenes como consecuencia del rápido desarrollo de las tecnologías de la información y la comunicación.

Actualmente, en nuestro Centro Universitario no se cuenta con un aula de computo adecuada para la cantidad de alumnos por grupo, en la materia de Química Orgánica, solo se dispone de pantalla de proyección y acceso a internet. En consecuencia, en este trabajo se hizo necesario la elaboración de material de apoyo, y se emplearon herramientas que pueden ser aplicadas en la enseñanza-aprendizaje de la Química Orgánica I, y en algunos temas de Química General, bajo las condiciones señaladas, es decir, haciendo uso de la computadora (incluso teléfonos inteligentes y tabletas) e Internet. Se diseñó un material educativo visual mediante la implementación de imágenes fijas y animadas, basados en 5 principios que se enumerarán más adelante, con el propósito de ayudar a los estudiantes a aprender y desarrollar habilidades para ayudar a explicar los conceptos, a través del apoyo a su pensamiento visoespacial (Wu y Shah, 2004), activando la creatividad, la motivación para el aprendizaje y la resolución de problemas.

En el aula se ha observado la efectividad que tiene implementar estas tecnologías, comprobando que los alumnos adquieren capacidades para el análisis y síntesis, así como seguridad en la comunicación y en la resolución de problemas (Gómez, 2006). Tal efectividad se debe a que las "TICs" atraen y conservan la atención del alumno, logrando que el proceso de enseñanza-aprendizaje sea efectivo, como se ha observado con el uso de herramientas, tales como; 1) Diapositivas dinámicas (Franco et al., 2010), 2) La "píldora del conocimiento" que se refiere al estudio del Equilibrio Químico, que permite la utilización de varios recursos como, imágenes, gráficos y esquemas, y además presenta como ventaja que el alumno puede utilizarla las veces que sea necesario (Maceiras et al., 2010), 3) La visualización y conversión de modelos moleculares bi y tri-dimensionales (Cabero, 2007; Marzocchi et al., 2012) para lo cual existen programas disponibles en diferentes sitios de Internet, entre ellos ChemSckech, RasMol, Chime, 3D Angles, Gabedit, y Jmol, 4) Simulaciones de procesos y prácticas de laboratorio virtuales (Cataldi, 2012).

Existen otros ejemplos interesantes de la aplicación de las TICS en la enseñanza-aprendizaje de la Química, como los descritos por Daza et al., (2009): I) Proyectos de alcance internacional, como Xperimania; II) El uso de simulaciones por ordenador, de procesos fisicoquímicos representados gráficamente; III) Material de apoyo y libro de texto digital; IV) Aplicaciones didácticas de los debates en línea, y V) Las plataformas de teleformación y el uso de entornos virtuales de aprendizaje. Aunque, 
paradójicamente, se han encontrado algunos casos donde se ha presentado resistencia al uso de las "TICs", particularmente en materias donde el recurso principal ha sido la memorización (Muñoz et al., 2011).

La enseñanza de la Química frecuentemente requiere de imágenes para facilitar la comprensión de conceptos, y la mejor prueba de esto es que los libros modernos de Química Orgánica tienen cada vez mayor número de ellas. Históricamente, en el campo de la Química Orgánica se han empleado diversos modelos para representar estructuras moleculares (Hoffmann y Torrence, 2004), abstraídas a partir de información obtenida experimentalmente y de cálculos teóricos, como en el caso de la estructura del benceno propuesta por Augusto Kekulé a mediados del siglo XIX. Recientemente hemos sido afortunados testigos de las primeras fotografías en alta definición obteniéndose imágenes detalladas de las estructuras de moléculas participantes en una reacción química donde se ha podido determinar los movimientos atómicos que ocurren en ella. Estas imágenes que muestran anillos de benceno, corresponden cabalmente al modelo propuesto por Kekulé. El estudio lo realizaron investigadores de las Universidades de Berkeley y del País Vasco (De Oteyza et. al., 2013). La figura 1 muestra el esquema de la reacción fotografiada por vez primera.

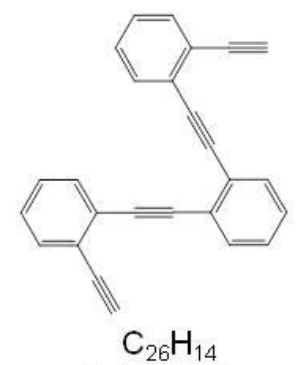

Materia prima

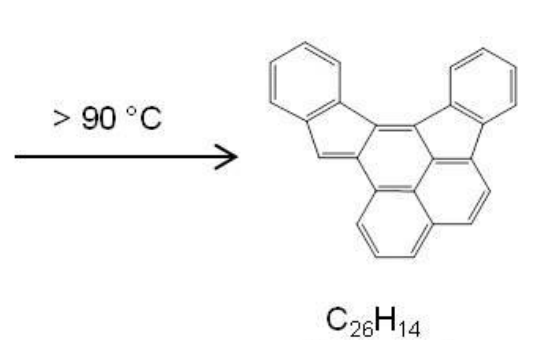

Producto 1

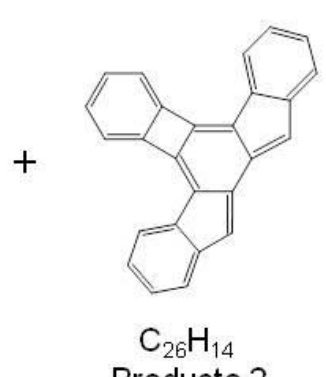

Producto 2

Fig. 1: Reacción química, de la que se obtuvo la primera fotografía en alta definición. La materia prima empleada fue un oligo-enediyne $\left(\mathrm{C}_{26} \mathrm{H}_{14}\right)$.

\section{METODOLOGÍA}

Este estudio fue realizado durante un periodo de 9 semestres comprendidos entre los ciclos 2008B-2013A, con un grupo de Química Orgánica I (denominado internamente D06), en el que compartieron aula alumnos de las licenciaturas de Químico-Farmacobiólogo, Química e Ingeniería Química del tercer y cuarto semestre. El docente encargado de llevar a cabo la prueba de esta estrategia de enseñanza, fue el mismo durante todo el estudio (el ciclo 2010A no se incluyó en el estudio, debido a que estuvo incapacitado por un problema de salud). La herramientas utilizadas durante los ciclos se dividió en tres tipos, la primera consistió en el uso en clases de imágenes 2D y 3D, la segunda, en la elaboración de juegos por parte de los alumnos y su exposición en el aula, y la tercera en la elaboración de videos educativos igualmente expuestos por ellos.

Las unidades del programa de aprendizaje incluidas en el estudio fueron: estructura y enlaces, enlaces covalentes polares: ácidos y bases, identificación de grupos funcionales, alcanos, cicloalcanos, tipos de reacciones orgánicas, alquenos: estructura y reactividad, y estereoquímica. Se utilizó pantalla de proyección, video proyector y acceso a internet (lo que denominamos "estilo con TICs"). Los alumnos tuvieron acceso al material utilizado a través de dispositivos USB y se les permitió utilizar sus teléfonos inteligentes y tabletas para tomar fotos y videos del material presentado en la clase.

El material didáctico utilizado como apoyo visual a los conceptos teóricos abordados durante el curso de Química Orgánica I durante los ciclos 2008B-2009B consistió en el uso de 104 imágenes a color, estáticas y en movimiento, compiladas de diferentes fuentes, algunas fueron creadas empleando programas comerciales (por ejemplo: Chem Draw Ultra 12.0, Perkín Elmer, para el trazo de mecanismos de reacción y moléculas en 2D y 3D). Este material se seleccionó cuidadosamente para resaltar los elementos implicados, que incluyeron conceptos importantes. Otro criterio significativo para la selección de las imágenes se basó en el hecho de que éstas deberían proporcionar la mayor cantidad de información posible, sin llegar a un exceso que pudiese tener efectos contrarios a los deseados, que permitiera al docente explicar el tema de manera clara y concisa. Para ello se tomaron en consideración cinco principios importantes de diseño (Wu y Shah, 2004): 1) Proporcionar múltiples representaciones y descripciones de los conceptos químicos, 2) Hacer visibles las conexiones referenciales entre los conceptos, 3) Representar la naturaleza dinámica e 
interactiva de la química, 4) Promover la transición entre 2D y 3D, y 5) Reducir la carga cognitiva en los estudiantes facilitándoles la información de forma explícita e integrada.

Respecto al primer tipo de herramienta utilizada, uno de los temas tratados con imágenes fue "estructura y enlace", donde se cubrieron conceptos básicos tales como: estructura atómica, regla del octeto, tipos de enlaces, teorías de enlace, orbitales moleculares, hibridación y electronegatividad. A continuación se muestran algunas imágenes empleadas para explicar los temas mencionados. Por ejemplo, en la figura 2 se explica la formación del doble enlace carbono-carbono, donde uno de los enlaces es sigma $(\sigma)$ y el otro es pi $(\pi)$ : el traslape frontal de dos orbitales híbridos $\mathrm{sp}^{2}$ da origen al enlace sigma $(\sigma)$, al tiempo que el traslape lateral de dos orbitales $p$ forma el enlace pi $(\pi)$ (McMurry, 2008; Yurkanis, 2008).

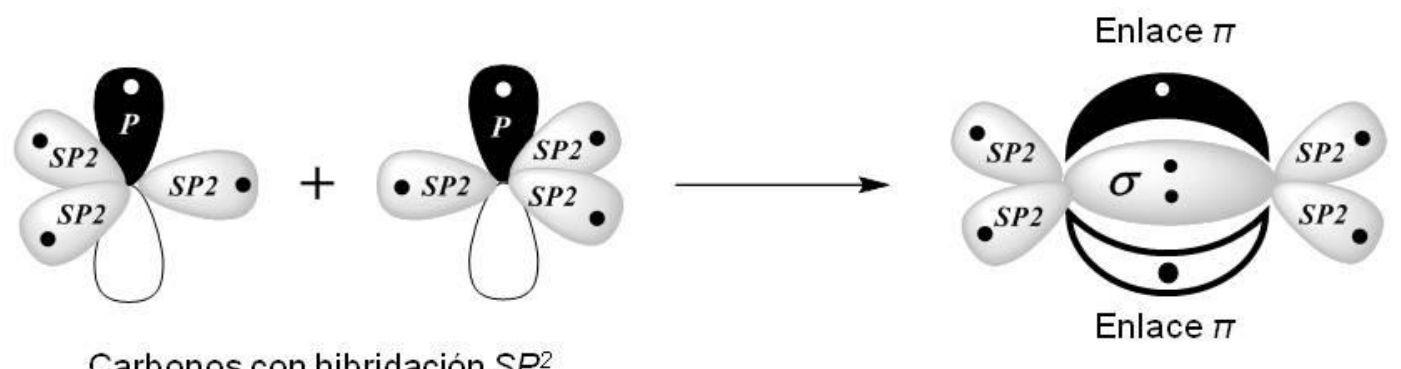

Carbonos con hibridación $\mathrm{SP}^{2}$

Doble enlace carbono-carbono

Fig. 2: Ayuda visual (molécula del etileno) para explicar la formación de un enlace doble carbono-carbono.

Otro ejemplo se muestra en la figura 3 que sirve de ayuda para mostrar longitudes y energías de diferentes tipos de enlaces. También se pueden explicar las magnitudes de los diferentes ángulos que se encuentran en moléculas que poseen carbonos con hibridación $\mathrm{sp}^{3}$ (metano), $\mathrm{sp}^{2}$ (eteno) y sp (etino). Así mismo, es posible observar la geometría de las mencionadas moléculas; tetraédrica en el metano, plana para el eteno y lineal en el caso del etino (Yurkanis, 2008; Wade, 2012).

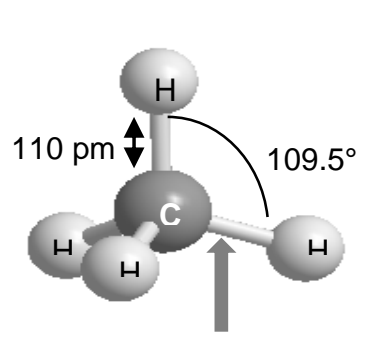

$105 \mathrm{Kcal} / \mathrm{mol}$

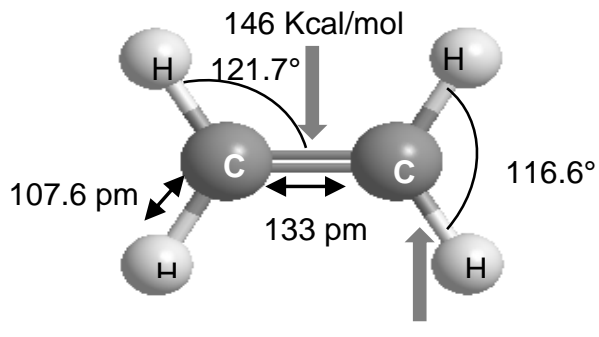

$106 \mathrm{Kcal} / \mathrm{mol}$

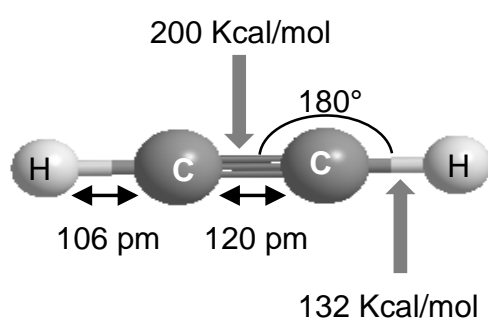

Metano

Eteno

Etino

Fig. 3: Ayuda visual para explicar la geometría molecular del metano, eteno y etino, respectivamente.

Con el fin de explorar las actividades lúdicas como estrategia de aprendizaje, a partir del 2010B se incluyeron juegos didácticos, elaborados por los alumnos en trabajo de equipo, utilizando los programas PowerPoint y Prezi. Los temas asignados, fueron impartidos previamente por el docente. Se dispusieron de 3 semanas para elaborar la totalidad de los juegos y de 20 minutos, para exponer cada uno de ellos en el aula. La dinámica consistió en proyectar en la pantalla la mayoría de los juegos, algunos se juegan de manera física (registrados en formato electrónico). En ambas modalidades el equipo con mayor número de respuestas correctas y en el menor tiempo, resulta ganador.

A continuación se describen algunos de los juegos: el denominado "sopa de letras" se utilizó en el tema de estructura y enlace; consiste en proyectar una imagen donde se localizan las posibles respuestas, de una serie de preguntas dirigidas a todos los equipos. Otro juego llamado "preguntas escondidas" se empleó en enlaces covalentes polares; consiste en encontrar algunos de los hexágonos escondidos en el salón de clase, cada hexágono tiene un número que corresponde a una pregunta y que puede ser contestada por el equipo poseedor, de lo contrario quedará libre para los otros equipos. El nombrado "moléculas ocultas" 
utilizado en alcanos y su estereoquímica; consiste en 3 rondas de preguntas (cada una de creciente grado de dificultad), donde se enfrentan 2 equipos y el capitán de cada uno de ellos selecciona el número de la pregunta a responder en cada ronda, esto se repite hasta completar el total de los equipos formados en el aula. Las respuestas correctas, permiten formar la molécula oculta. "El crucigrama" con el contenido de cicloalcanos y su estereoquímica; se basa en las reglas conocidas. En el "jeopardy" para perspectivas de las reacciones orgánicas; cada equipo selecciona al azar un número y se procede a responder las preguntas correspondientes. Con la "lotería tradicional mexicana" se trató el tema de alquenos: estructura y reactividad; donde cada equipo toma una carta, gana el equipo que llene primero su carta. En la baraja está el nombre y la estructura, mientras que en la carta se encuentran solamente estructuras de moléculas 0 intermediarios (por ejemplo, carbocationes). A continuación se muestran imágenes (Fig. 4) de algunos de los juegos descritos.

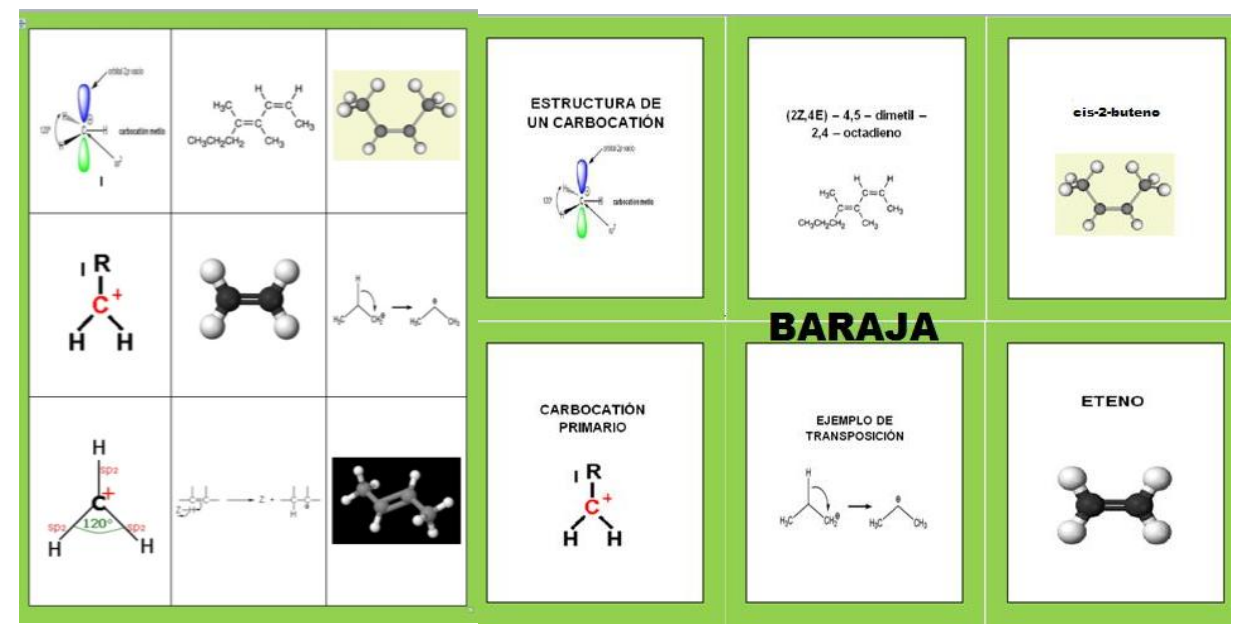

a)

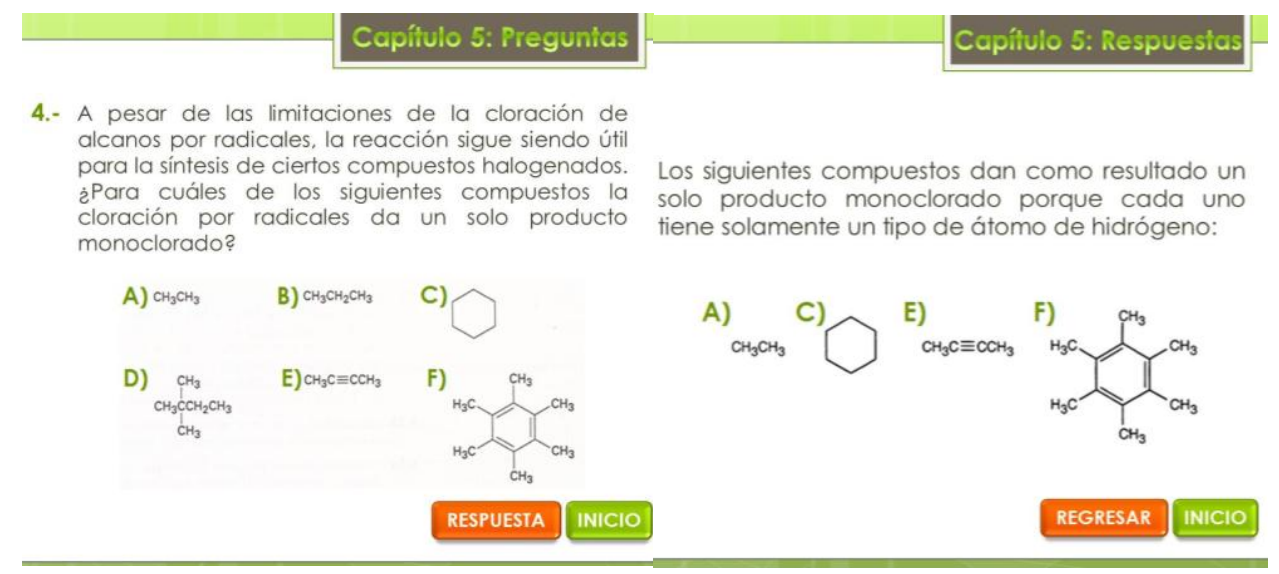

b)

Fig. 4: a) Lotería tradicional mexicana, se muestra una carta y parte de la baraja de la lotería, b) Jeopardy, se muestra una pregunta con su respectiva respuesta.

Del 2012A al 2013A, ciclos en los que además se incluyeron videos educativos también elaborados por los alumnos y con libertad de elección en la forma de la presentación, que lleva aproximadamente un mes en la realización y se disponen de 15 minutos para exposición en el aula. A continuación se mencionan algunos temas tratados con esta herramienta: estructura atómica, enlaces químicos e hibridación; con imágenes en movimiento y narración, que permiten observar las características estructurales, cargas formales y resonancia; se relatan en forma de noticiero donde se describen las reglas implicadas, ácidos y bases; se utilizaron dibujos animados explicando todos los conceptos relacionados, grupos funcionales; se hizo una video grabación de moléculas elaboradas por los alumnos, donde se da una descripción que permite su identificación. alcanos y cicloalcanos; se narra como un partido de futbol, en donde se muestran y describen las estructuras además de sus propiedades, y estereoquímica; se trató con marionetas, de manera humorística se hace énfasis en el concepto de quiralidad. En la figura 5 se muestran imágenes de algunos de los videos descritos. 


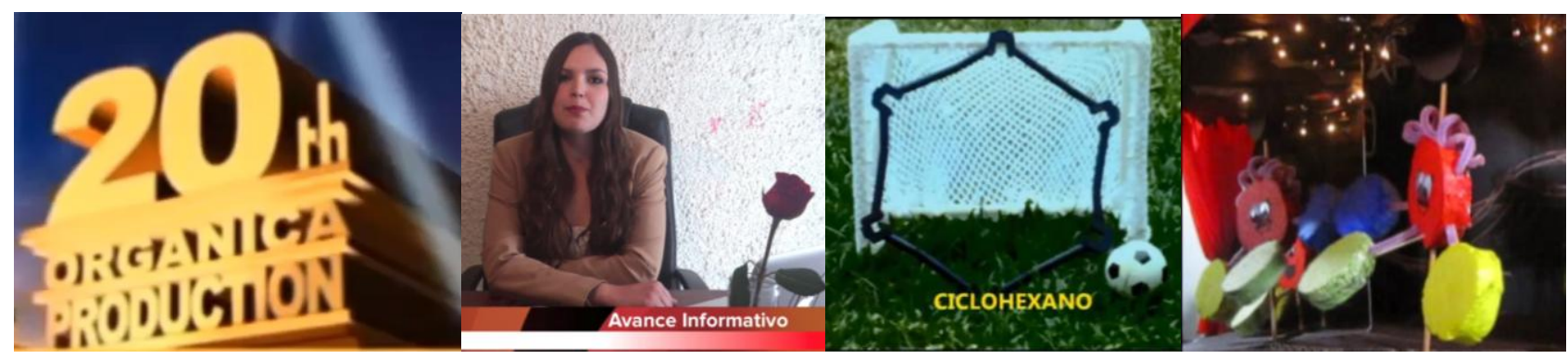

Fig. 5: Imágenes de algunos videos educativos empleados, como parte del estudio objeto de este trabajo.

Una ventaja adicional de utilizar estas herramientas consiste en el ahorro de tiempo en la exposición de los temas, el cual es utilizado en talleres de resolución de problemas y en la presentación de trabajos de investigación de los alumnos, lo cual optimiza su aprendizaje, y es reflejado en los resultados.

\section{RESULTADOS}

En la Tabla 1 se muestra los resultados obtenidos en la evaluación de nueve ciclos escolares comprendidos del periodo 2008B al 20013A con un total de 296 alumnos, 251 Aprobados (A) y 45 No Aprobados (NA); de los alumnos en la categoría NA, la mayoría desertaron en el transcurso del ciclo. A estos últimos, el Sistema Integral de Información y Administración Universitaria (SIIAU) de nuestra Institución, los considera con calificación igual a cero lo que impacta negativamente en el promedio y aumenta la desviación estándar.

Con los datos de las dos últimas columnas de la Tabla 1, se elaboró la gráfica de porcentaje de aprobación que se muestra en la figura 6 , donde se puede apreciar un ascenso en el porcentaje de alumnos aprobados $(\% A)$. Con los datos de la tercera columna de la Tabla 1, se elaboró la gráfica de promedios que se muestra en la figura 7, donde se puede apreciar un incremento en el promedio de calificación.

Observando la tendencia creciente de las medias o promedios de calificaciones, se procedió a realizar un análisis de varianza para comprobar o descartar que las medias sean significativamente diferentes, considerando que cada ciclo escolar es un "tratamiento" dentro del "diseño completamente aleatorizado", cuyo modelo es: $Y_{i j}=\mu+\tau_{i}+\varepsilon_{i j}$, en el cual la hipótesis nula es Ho: todos los efectos de los tratamientos son iguales, $\tau_{1}=\tau_{2}=\ldots=\tau_{t}$, y la hipótesis alternativa es $\mathrm{Ha}=\mathrm{al}$ menos un efecto de un tratamiento es diferente a los demás, $\tau_{i} \neq \tau_{j}$ (Infante y Zárate, 1990). Los resultados obtenidos se muestran en la Tabla 2; de acuerdo a la Fo calculada (Fo > F), debe rechazarse Ho con un nivel de significancia de 0.01, y entonces aceptar que al menos un tratamiento es diferente.

Tabla 1: Calificaciones promedio y datos de aprobación

\begin{tabular}{|c|c|c|c|c|c|}
\hline Ciclo & $\begin{array}{c}\text { Número de } \\
\text { alumnos }\end{array}$ & $\begin{array}{c}\text { Calificación } \\
\text { Promedio }\end{array}$ & $\begin{array}{c}\text { Desviación } \\
\text { Estándar }\end{array}$ & $\begin{array}{c}\text { Aprobados } \\
(\mathrm{A})\end{array}$ & $\begin{array}{c}\text { No Aprobados } \\
(\mathrm{NA})\end{array}$ \\
\hline $2008 \mathrm{~B}$ & 25 & 44.60 & 37.79 & 15 & 10 \\
\hline 2009A & 35 & 59.71 & 31.10 & 28 & 7 \\
\hline $2009 \mathrm{~B}$ & 35 & 56.77 & 27.77 & 27 & 8 \\
\hline 2010B & 32 & 60.22 & 27.17 & 27 & 5 \\
\hline 2011A & 30 & 58.37 & 30.80 & 24 & 6 \\
\hline 2011B & 30 & 67.60 & 24.63 & 27 & 3 \\
\hline 2012A & 35 & 73.20 & 24.02 & 32 & 3 \\
\hline 2012B & 36 & 74.47 & 20.30 & 34 & 2 \\
\hline 2013A & 38 & 79.87 & 16.93 & 37 & 1 \\
\hline Total de alumnos & 296 & --- & --- & 251 & 45 \\
\hline Promedio global & --- & 64.78 & 28.28 & -- & -- \\
\hline
\end{tabular}




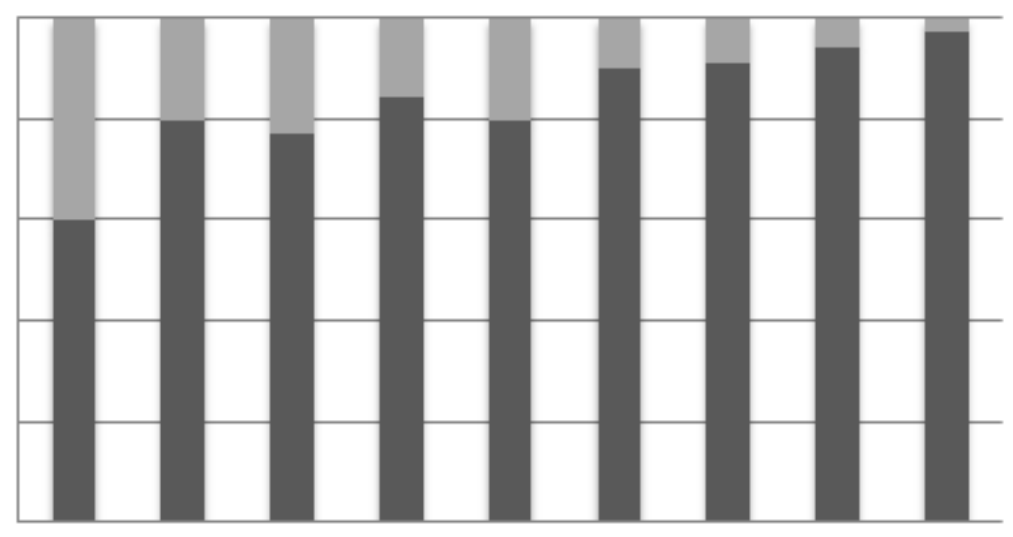

Fig. 6: Gráfica de porcentaje de aprobación.

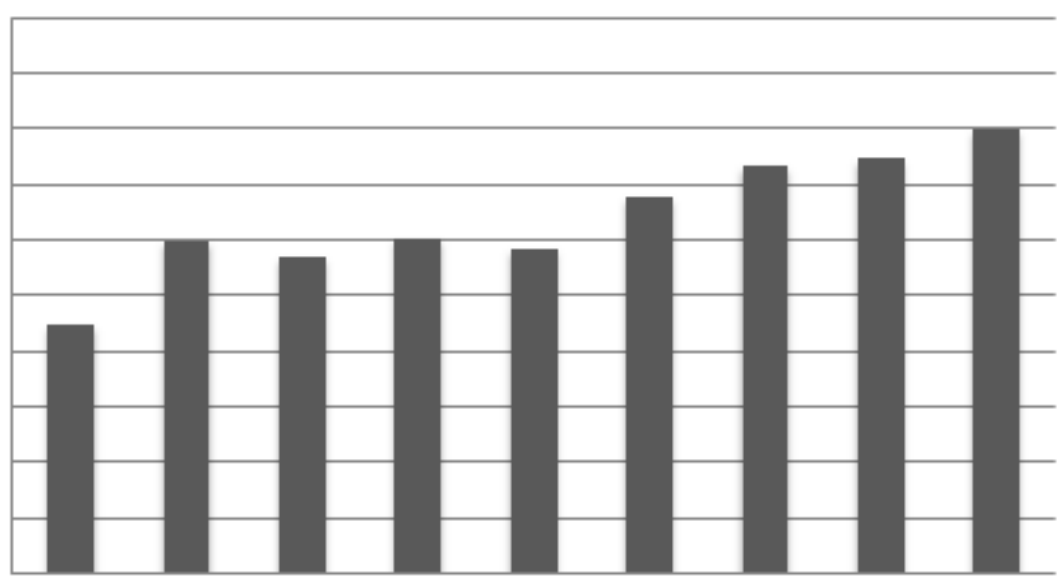

Fig. 7: Gráfica de medias o promedios de calificaciones.

Tabla 2: Análisis de varianza de calificaciones

\begin{tabular}{|l|c|c|c|c|c|}
\hline $\begin{array}{c}\text { Fuente de } \\
\text { Variación }\end{array}$ & $\begin{array}{c}\text { Grados de } \\
\text { Libertad }\end{array}$ & $\begin{array}{c}\text { Suma de } \\
\text { Cuadrados }\end{array}$ & $\begin{array}{c}\text { Cuadrado } \\
\text { Medio }\end{array}$ & Fo & $F(8 / 287, \alpha=0.99)$ \\
\hline Tratamientos & 8 & 29976.30 & 3747.037 & 5.22 & 2.5 \\
\hline Error & 287 & 205985.86 & 717.721 & & \\
\hline Total & 295 & 235962.16 & & & \\
\hline
\end{tabular}

Para encontrar cuáles tratamientos ocasionan el rechazo de Ho en el análisis de varianza, se realizó la "técnica de contrastes". Aquí, la hipótesis nula $\mathrm{Ho}$, es $Q=0$, es decir, el contraste $(Q)$ es igual a cero, en oposición a Ha, donde $Q \neq 0$. El estadístico de prueba es la variable to (Infante y Zárate, 1990).

De las múltiples comparaciones que pueden hacerse entre 9 tratamientos, se tomó la decisión de agrupar los tratamientos en tres tríadas y compararlas entre pares de ellas. Este criterio de agrupamiento obedeció a que las metodologías de los tratamientos de cada triada fue equivalente entre sí, pero con algunas diferencias de metodología entre tratamientos de las otras tríadas, según se describió en la Sección de Metodología. Los ciclos escolares se agruparon según se especifica en la Tabla 3.

Tabla 3: Agrupamiento de ciclos escolares para pruebas de contraste

\begin{tabular}{|c|c|l|}
\hline Grupos & Tríadas & Herramientas \\
\hline I & 08B, 09A, 09B & Imágenes 2D y 3D, estáticas y dinámicas \\
\hline II & 10B, 11A, 11B & Incluye dinámica de juegos, elaboración y participación de los alumnos \\
\hline III & $12 \mathrm{~A}, 12 \mathrm{~B}, 13 \mathrm{~A}$ & Incluye vídeos, elaboración y participación de los alumnos \\
\hline
\end{tabular}


Con esta consideración, $Q=1 / 3\left(\tau_{1}+\tau_{2}+\tau_{3}\right)-1 / 3\left(\tau_{4}+\tau_{5}+\tau_{6}\right)$, donde $\left(\tau_{1}, \tau_{2}, \tau_{3}\right)$ son las medias del primer grupo y $\left(\tau_{4}, \tau_{5}, \tau_{6}\right)$ son las medias del segundo grupo. A los coeficientes del tercer grupo que es excluido en la comparación se les asigna un valor igual a cero, es decir, $\mathrm{Q}=1 / 3\left(\tau_{1}+\tau_{2}+\tau_{3}\right)-1 / 3\left(\tau_{4}+\tau_{5}+\tau_{6}\right)+0\left(\tau_{7}+\tau_{8}+\right.$ $\left.\tau_{9}\right)$. En la Tablas 4, 5 y 6 se muestran los resultados obtenidos en las pruebas de contrastes de los pares de grupos I y II, I y III, II y III, respectivamente. Cada contraste individual $Q_{i}$ se obtiene mediante $Q_{i}=\left(C_{i}\right)\left(\tau_{i}\right)$, y el valor del contraste $Q$ es el sumatorio $\Sigma Q_{i}$.

Tabla 4: Contraste entre tratamientos de los grupos I y II

\begin{tabular}{|l|r|r|r|r|r|r|r|r|r|r|}
\hline & \multicolumn{3}{|c|}{ Grupo A } & \multicolumn{3}{c|}{ Grupo B } & & \\
\hline Tratamiento (ciclos) & \multicolumn{1}{|c|}{ 08B } & \multicolumn{1}{c|}{ 09A } & \multicolumn{1}{c|}{ 09B } & \multicolumn{1}{c|}{ 10B } & \multicolumn{1}{c|}{$11 \mathrm{~A}$} & \multicolumn{1}{c|}{$11 \mathrm{~B}$} & \multicolumn{1}{c|}{$12 \mathrm{~A}$} & \multicolumn{1}{c|}{$12 \mathrm{~B}$} & \multicolumn{1}{c|}{$13 \mathrm{~A}$} & Suma \\
\hline Coeficiente, $\mathrm{C}_{\mathrm{i}}$ & 0.33 & 0.33 & 0.33 & -0.33 & -0.33 & -0.33 & 0.00 & 0.00 & 0.00 & 0.00 \\
\hline Media, $\tau_{i}$ & 44.60 & 59.71 & 56.77 & 60.22 & 58.37 & 67.60 & 73.20 & 74.47 & 79.87 & -- \\
\hline Tamaño muestra, $\mathrm{n}_{\mathrm{i}}$ & 25 & 35 & 35 & 32 & 30 & 30 & 35 & 36 & 38 & 296 \\
\hline Coeficientes $^{2}, \mathrm{C}_{\mathrm{i}}{ }^{2}$ & 0.1111 & 0.1111 & 0.1111 & 0.1111 & 0.1111 & 0.1111 & 0.00 & 0.00 & 0.00 & 0.6667 \\
\hline $\mathrm{C}_{\mathrm{i}}{ }^{2} / \mathrm{n}_{\mathrm{i}}$ & 0.0044 & 0.0032 & 0.0032 & 0.0035 & 0.0037 & 0.0037 & 0.00 & 0.00 & 0.00 & 0.0217 \\
\hline Contrastes $\mathrm{Q}_{\mathrm{i}}$ & 14.867 & 19.903 & 18.923 & -20.073 & -19.457 & -22.533 & 0.00 & 0.00 & 0.00 & -8.3700 \\
\hline
\end{tabular}

Tabla 5: Contraste entre tratamientos de los grupos I y III

\begin{tabular}{|c|c|c|c|c|c|c|c|c|c|c|}
\hline \multirow[b]{2}{*}{ Tratamiento (ciclos) } & \multicolumn{3}{|c|}{ Grupo A } & \multirow[b]{2}{*}{ 10B } & \multirow[b]{2}{*}{$11 \mathrm{~A}$} & \multirow[b]{2}{*}{ 11B } & \multicolumn{3}{|c|}{ Grupo C } & \multirow[b]{2}{*}{ Suma } \\
\hline & 08B & 09A & 09B & & & & $12 \mathrm{~A}$ & $12 \mathrm{~B}$ & $13 \mathrm{~A}$ & \\
\hline Coeficiente, $\mathrm{C}_{\mathrm{i}}$ & 0.33 & 0.33 & 0.33 & 0.00 & 0.00 & 0.00 & -0.33 & -0.33 & -0.33 & 0.00 \\
\hline Media, $\tau_{i}$ & 44.60 & 59.71 & 56.77 & 60.22 & 58.37 & 67.60 & 73.20 & 74.47 & 79.87 & -- \\
\hline Tamaño muestra, $n_{i}$ & 25 & 35 & 35 & 32 & 30 & 30 & 35 & 36 & 38 & 296 \\
\hline Coeficientes $^{2}, \mathrm{C}_{\mathrm{i}}^{2}$ & 0.1111 & 0.1111 & 0.1111 & 0.00 & 0.00 & 0.00 & 0.1111 & 0.1111 & 0.1111 & 0.6667 \\
\hline $\mathrm{C}_{\mathrm{i}}^{2} / \mathrm{n}_{\mathrm{i}}$ & 0.0044 & 0.0032 & 0.0032 & 0.00 & 0.00 & 0.00 & 0.0032 & 0.0031 & 0.0029 & 0.0200 \\
\hline Contrastes $Q_{i}$ & 14.867 & 19.903 & 18.923 & 0.00 & 0.00 & 0.00 & -24.400 & -24.824 & -26.623 & -22.153 \\
\hline
\end{tabular}

Tabla 6: Contraste entre tratamientos de los grupos II y III

\begin{tabular}{|c|c|c|c|c|c|c|c|c|c|c|}
\hline & & & & \multicolumn{3}{|c|}{ Grupo B } & \multicolumn{3}{|c|}{ Grupo C } & \\
\hline Tratamiento (ciclos) & $08 \mathrm{~B}$ & $09 \mathrm{~A}$ & 09B & 10B & $11 \mathrm{~A}$ & $11 \mathrm{~B}$ & $12 \mathrm{~A}$ & $12 \mathrm{~B}$ & $13 \mathrm{~A}$ & Suma \\
\hline Coeficiente, $\mathrm{C}_{\mathrm{i}}$ & 0.00 & 0.00 & 0.00 & 0.33 & 0.33 & 0.33 & -0.33 & -0.33 & -0.33 & 0.00 \\
\hline Media, $\tau_{i}$ & 44.60 & 59.71 & 56.77 & 60.22 & 58.37 & 67.60 & 73.20 & 74.47 & 79.87 & -- \\
\hline Tamaño muestra, $n_{i}$ & 25 & 35 & 35 & 32 & 30 & 30 & 35 & 36 & 38 & 296 \\
\hline Coeficientes $^{2}, \mathrm{C}_{\mathrm{i}}^{2}$ & 0.00 & 0.00 & 0.00 & 0.1111 & 0.1111 & 0.1111 & 0.1111 & 0.1111 & 0.1111 & 0.6667 \\
\hline $\mathrm{C}_{\mathrm{i}}^{2} / \mathrm{n}_{\mathrm{i}}$ & 0.00 & 0.00 & 0.00 & 0.0035 & 0.0037 & 0.0037 & 0.0032 & 0.0031 & 0.0029 & 0.0201 \\
\hline Contrastes $Q_{i}$ & 0.00 & 0.00 & 0.00 & 20.073 & 19.457 & 22.533 & -24.400 & -24.824 & -26.623 & -13.784 \\
\hline
\end{tabular}

El estadístico to se calcula mediante $\left(\Sigma Q_{\mathrm{i}} / S_{Q}\right)$ donde $S_{Q}$ es la desviación estándar del contraste; a su vez, la varianza del contraste se calcula mediante $S_{Q}{ }^{2}=\operatorname{CME}\left(\Sigma \mathrm{C}_{i}{ }^{2} / \mathrm{n}_{\mathrm{i}}\right)$, siendo CME el Cuadrado Medio del Error, estadístico previamente obtenido en el análisis de varianza, y que es el estimador de la varianza $\left(\sigma^{2}\right)$, con sus respectivos grados de libertad (Infante y Zárate, 1990). En la Tabla 7 se muestran los valores de las variables aquí mencionadas, así como el valor de la Distribución $t$, que es función de cada to, con 287 grados de libertad. Como se aprecia en la última columna de la Tabla 7, en las tres comparaciones debe ser rechazada $\mathrm{Ho}$, lo que implica que cada grupo de tratamientos es diferente.

Tabla 7: Valores de to y conclusiones de las pruebas de contraste

\begin{tabular}{|c|c|c|c|c|c|l|}
\hline Grupos & CME & $S_{Q}{ }^{2}$ & $S_{Q}$ & to & Dist t & \multicolumn{1}{|c|}{ Conclusión } \\
\hline I y II & 717.721 & 15.555 & 3.944 & -2.122 & 0.9827 & Se rechaza Ho, 98.27\% de certidumbre \\
\hline I y III & 717.721 & 14.339 & 3.787 & -5.850 & 1.000 & Se rechaza Ho, 100\% de certidumbre \\
\hline II y III & 717.721 & 14.401 & 3.795 & -3.632 & 0.9998 & Se rechaza Ho, 99.98\% de certidumbre \\
\hline
\end{tabular}




\section{DISCUSIÓN}

Los resultados cuantitativos obtenidos en este estudio (Tabla 1) reflejan que, bajo las condiciones descritas en la metodología, el curso de Química Orgánica I sujeto a evaluación mostró una notable mejoría, principalmente en lo que se refiere a alumnos que aprobaron el curso (A), y como consecuencia de ello, en el aumento del promedio de calificaciones, pues como se mencionó en la sección de Resultados, la mayoría de los alumnos No Aprobados (NA) tienen asignada una calificación igual a cero.

Por otra parte, los resultados del análisis estadístico mediante la "técnica de contrastes" (Tablas 2-7), muestran que a medida que los alumnos participan del trabajo colaborativo, elaborando y presentando en clase sus propios materiales educativos, incrementan su rendimiento académico y su interés por la materia, pasando de una actitud pasiva a una conducta activa, ya que se logra captar mejor la atención de los alumnos, y por ende, una mayor comprensión de los temas ofrecidos, y finalmente se consigue un punto de quiebre en la tradicional "aversión" hacia la Química, consecuencia de deficiencias acumuladas durante grados anteriores.

Parte importante de este trabajo fue la adecuada presentación de la información visual, siguiendo ciertos principios de diseño y selección del material utilizado, que permitió activar todos los procesos cognitivos posibles, llegando a una definición de cada concepto de manera interactiva mediante una explicación del docente y una deducción por parte de los alumnos. Este enfoque es importante dado que hace uso de las tecnologías más actuales en cuanto a la presentación de información, por ejemplo con las computadoras personales, los teléfonos inteligentes y tabletas, dispositivos con los que el estudiante actual se siente más cómodo, que con un cuaderno y libro tradicional. Debido a esto, consideramos que este enfoque forma parte de nuestro presente y futuro, y debemos aprovechar sus fortalezas y atenuar sus debilidades (Daza et al., 2009).

\section{CONCLUSIONES}

El trabajo que aquí se presenta podría ser útil para docentes universitarios de Países en vías de desarrollo de reciente incursión en el uso de las "TICs". Es una experiencia docente en la enseñanza-aprendizaje de la Química Orgánica donde se aplicó una metodología basada en el uso de las "TICs" para la facilitar la formación de nuestros estudiantes, obteniéndose las siguientes conclusiones principales:

1. El resultado principal al utilizar esta metodología, consistió en lograr un impacto positivo en la eficiencia terminal del curso y, por consiguiente, un uso más eficiente de los recursos institucionales destinados a la educación. Debe considerarse que cada día se incrementa el acceso a tecnologías como internet, libros electrónicos, computadoras y tabletas (entre otras), que impactan positivamente en el rendimiento académico de los alumnos.

2. Se facilitó la comprensión de los conceptos, debido a que "una imagen dice más que mil palabras". Esto es particularmente aplicable, cuando se emplean imágenes en movimiento (videoclips y/o películas) ya que estas describen fenómenos dinámicos y además se requiere menos tiempo para reflexionar.

3. El material audiovisual puede ser consultado de manera extra-clase por los alumnos a través de dispositivos USB, teléfonos inteligentes, tabletas, y, también en la plataforma Moodle de nuestra universidad, en el último ciclo escolar.

4. La implementación de las "TICs" objeto de este trabajo didáctico, constituye una herramienta pedagógica de suma utilidad para la enseñanza de la Química Orgánica I, considerando que dicha disciplina posee su propio lenguaje basado de manera importante en símbolos, que se auxilian con el uso de numerosas imágenes.

5.- A medida que incrementa el trabajo colaborativo entre los alumnos, mayor es el impacto en el desarrollo de los procesos cognitivos, lo que se refleja en la mejora de los resultados de sus evaluaciones.

\section{AGRADECIMIENTOS}

Osvaldo Camacho Castillo, Depto. de Matemáticas del CUCEI; Cesar Ricardo Cortéz Álvarez, Depto. de Farmacobiología del CUCEl; Juan Humberto Pérez López, Depto. de Ing. Química del CUCEl; Rafael Alejandro Ramírez Gómez, Coordinación de Tecnologías para el Aprendizaje del CUCEl; Hilda Patricia Ortiz Villaseñor, Depto. de Comunicación y Aprendizaje, Preparatoria 2. 


\section{REFERENCIAS}

Bekerman, D. G. y Dankner L. A., La Pareja Pedagógica en el Ámbito Universitario, Un Aporte a la Didáctica Colaborativa, Formación Universitaria, 3(6), 3-8 (2010).

Cabero, A. J., Las TICs en la enseñanza de la química: aportaciones desde la Tecnología Educativa, Química: vida y progreso, En BODALO, A. y otros (eds), Murcia, España (2007).

Carnoy, M., Las TIC en la Enseñanza: Posibilidades y Retos. En: Lección inaugural del curso académico 2004-2005 (en línea), (2004). http://www.uoc.edu/inaugural04/esp/index.html. Acceso: 5 de enero de 2012.

Cataldi, Z., Dominighini, C., Chiarenza, D. y Lage, F. J., TICs en la enseñanza de la Química: Propuesta de Evaluación Laboratorios Virtuales de Química ( $L V Q s)$, Revista Iberoamericana de Educación en Tecnología y Tecnología en Educación, No. 7, 50-59 (2012).

Daza, P. E. P. y otros 9 autores, Experiencias de enseñanza de la química con el apoyo de las TIC, Educación Química (en línea), UNAM, XX(3), 320-329 (2009).

De Oteyza, D. G. y otros 11 autores, Direct Imaging of Covalent Bond Structure in Single-Molecule Chemical Reactions, Science, 340(6139), 1434-1437 (2013).

Franco M. F., Cruz L. O., Díaz G. M., Evaluación de la implantación de un nuevo recurso didáctico diapositivas dinámicas- en la asignatura de Química Orgánica en la Licenciatura de Farmacia, ARH Pharmacéutica, 51(1), 191-200 (2010).

Gómez, D. A., Incorporación de las TICs en el aula de química, STUDIOSITAS.BOGOTA (COLOMBIA), 1(1), 18-22 (2006).

Hernández R. S., El Modelo Constructivista con las Nuevas Tecnologías, Aplicado en el Proceso de Aprendizaje, Rev. de Universidad y Sociedad del Conocimiento, 5(2), 26-35 (2003).

Hoffmann, R. y Torrence, V., Química imaginada: Reflexiones sobre la ciencia, 17-27, Fondo de Cultura Económica, México (2004).

Infante G. S. y Zárate L. G. P., Métodos Estadísticos: Un enfoque interdisciplinario, $2^{2}$ edición, 417-424, 435439, Editorial Trillas S. A. de C. V., México, D. F., México (1990).

Jiménez V. G., Llitjós V. A., Recursos didácticos audiovisuales en la enseñanza de la química: una perspectiva histórica, Educación Química, 17(2), 158-163 (2006).

Khvilon E., et al., Las Tecnologías de la Información y la Comunicación en la Formación Docente - Guía de Planificación, UNESCO, Ediciones Trilce, Montevideo, Uruguay (2004).

Maceiras R., Cancela A. y Goyanes V., Aplicación de Nuevas Tecnologías en la Docencia Universitaria, Formación Universitaria, 3(1), 21-26 (2010).

Marzocchi, V. y otros 4 autores, Incorporación de TICs de modelado molecular en la enseñanza universitaria de la Química, Rev. Iberoam. de Educ. en Tecnología y Tecnología en Educación, No. 8, 9-15 (2012).

McMurry, J., Química Orgánica, 7ª edición, 1-40, Cengage Learning, México, D.F. México (2008).

Miranda, A., Santos, G. y Stipcich, S., Algunas Características de Investigaciones que Estudian la Integración de las TIC en la Clase de Ciencia, Rev. Electr. de Invest. Educ., 12(2), 1-24, (2010).

Moreno, M. E., Las TIC y el Desarrollo del Aprendizaje en Educación Inicial, Rev. Electr. de Humanidades, Educ. y Comunic. Social, $1^{a}$ edición, año 1, 1-11 (2006).

Muñoz C. J. M., Córdova J. A., Priego H., Dificultades y facilidades para el desarrollo de un proceso de innovación educativa con base en las TICs, Formación Universitaria, 5(1), 3-12 (2012).

Prensky, M., Teaching Digital Natives: Partnering for Real Learning, $1^{\text {a }}$ edition, 1-8, Corwin Press, California, USA (2010).

Wade, L. G. Jr., Química Orgánica, Vol. 1, 7ª̣ edición, 6-10 y 40-56, Pearson Educación de México, S. A. de C. V., México (2012).

Wu, H. K., Shah, P., Exploring Visuospatial Thinking in Chemistry Learning, Science Education, 88(3), 465492 (2004).

Yurkanis, B. P., Química Orgánica, 5a Edición, 28-43, Pearson Educación de México, S. A. de C. V., Naucalpan de Juárez, México (2008). 